\title{
Theory of the angular magnetoresistance in CPP spin valves
}

\author{
Daniel Huertas-Hernando, Gerrit E. W. Bauer and Yu. V. Nazarov \\ Department of Applied Physics and Delft Institute of Microelectronics and Submicrontechnology, \\ Delft University of Technology, Lorentzweg 1, 2628 CJ Delft, The Netherlands
}

(November 21, 2018)

\begin{abstract}
The resistance of CPP spin valve is a continuous function of the angle $\theta$ between the magnetizations of both ferromagnets. We use the cicuit theory for non-collinear magnetoelectronics to compute the angular magnetoresistance of CPP spin valves taking the spin accumulation in the ferromagnetic layers into account.
\end{abstract}

The magnetoresistance of multilayers in the so-called current perpendicular to the plane (CPP) configuration first studied by the Michigan State University and Philips groups [1], is still an active field of research. Most studies have been focused on collinear, parallel and antiparallel, magnetic configurations. There are several papers that have investigated also non-collinear transport [2,3]. The circuit theory of mesoscopic transport [4 has been applied to describe transport in non-collinear magnetic structures [5]. Interesting phenomena like spin precession effects on the induced spin accumulation have been found by solving the diffusion equation for CPP spin valves [6]. An important concept in the theory of non-collinear spin transport is the so-called mixing conductance $G^{\uparrow \downarrow}[5]$ which is closely related to the current-induced spin-torque [7]. In this paper we extend our previous theoretical work on the total conductance of CPP ferromagnet-normal metal-ferromagnet $(\mathrm{F}|\mathrm{N}| \mathrm{F})$ spin valves, taking into account the spin-accumulation in the ferromagnets 87 .

In our model two ferromagnetic layers (F) are connected to a normal layer through metallic contact regions or interfaces (C). The system may split up into reservoirs, resistors and nodes (Fig.1) [9]. Two fictitious nodes connect each F-layer with a contact region $\mathrm{C}$, where $\mathrm{F}$ is represented by the spin-up and spin-down conductances and $\mathrm{C}$ is described by four spin-dependent conductances. We assume that no spin-flip processes occur neither at the interfaces nor in the bulk layers. The resistance in the normal metal node is also disregarded. Associated with each node is a $2 \times 2$ distribution matrix in spin space which represents charge and spin-accumulation. The spin accumulation in the ferromagnetic (F-C) node is taken to be parallel to the magnetization $\overrightarrow{\mathbf{m}}_{1 / 2}$ of the ferromagnet. The spin and charge currents are then completely determined by the relative orientation of the magnetization directions in the ferromagnets $\left(\overrightarrow{\mathbf{m}}_{1} \cdot \overrightarrow{\mathbf{m}}_{2}=\cos \theta\right)$, the spin-conductances associated with the ferromagnetic layers $\left(G_{F}^{\uparrow}, G_{F}^{\downarrow}\right)$ and the contact conductances $\left(G_{c}^{\uparrow}, G_{c}^{\downarrow}, G^{\uparrow \downarrow}\right)$. According to first-principles calculations [10] $\operatorname{Re} G^{\uparrow \downarrow} \gg \operatorname{Im} G^{\uparrow \downarrow}$ so in the following we disregard the imaginary part. For the symmetric case, in which the conductances are equal for both interfaces and also for both F-layers, the total conductance reads

$$
G^{T}(\theta)=\frac{1}{2}\left\{\frac{\left[G_{F} G_{c}^{\uparrow} G_{c}^{\downarrow}+G_{c} G_{F}^{\uparrow} G_{F}^{\downarrow}\right] 2 \operatorname{Re} G^{\uparrow \downarrow}(1+\cos \theta)+\left[4 G_{c}^{\uparrow} G_{c}^{\downarrow} G_{F}^{\uparrow} G_{F}^{\downarrow}\right](1-\cos \theta)}{\left[G_{c}^{\uparrow} G_{c}^{\downarrow}+G_{F}^{\uparrow} G_{F}^{\downarrow}+G_{F}^{\uparrow} G_{c}^{\downarrow}+G_{F}^{\downarrow} G_{c}^{\uparrow}\right] 2 \operatorname{Re} G^{\uparrow \downarrow}(1+\cos \theta)+\left[G_{F} G_{c}^{\uparrow} G_{c}^{\downarrow}+G_{c} G_{F}^{\uparrow} G_{F}^{\downarrow}\right](1-\cos \theta)}\right\}
$$

Eq. (11) reduces to well-known collinear expressions for $\theta=0$ and $\theta=\pi$. When $G_{F} \rightarrow \infty$, Eq.(24) in Ref. [6] $G^{T}(\theta)$ is recovered. In the absence of a spin-dependent interface resistance, i.e. $P_{c}=0\left(G_{c}^{\uparrow}=G_{c}^{\downarrow}\right), G^{T}(0)-G^{T}(\pi)$ is governed exclusively by the spin accumulation of the bulk F-layers.

Using Eq. (1), we obtain the following expression for the normalized angular-MR (NAMR)

$$
\operatorname{NAMR}(\theta)=\left(\frac{G^{T}(\theta)-G^{T}(\theta=0)}{G^{T}(\theta=\pi)-G^{T}(\theta=0)}\right)\left(\frac{G^{T}(\theta=\pi)}{G^{T}(\theta)}\right)=\frac{1-\cos ^{2} \theta / 2}{\chi \cos ^{2} \theta / 2+1}
$$

with

$$
\chi=\operatorname{Re} \eta\left[\frac{1}{1-p_{c}^{2}}+\frac{\left(G_{c} / G_{F}\right)}{1-p_{F}^{2}}\right]-1 .
$$

From Eq. (2a), we see that the NAMR depends on the angle $\theta$ between the magnetization of both ferromagnets and on the parameter $\chi$, which depends on the contact and F-layer conductance parameters. Similar expression for the NMAR have been obtained using the Kubo formalism [3]. They use a different model that forbids the direct comparison of the results. In their case, no resistance was associated with the interfaces between layers. Rather, the effect arises from the finite resistance of the normal layer and spin-dependent resistance of the ferromagnets. 
The parameters of the 2-channel series resistor model for collinear configurations have been accurately determined from CPP magnetoresistance experiments. For future comparison of our expression with NAMR experimental data, the quantities $p_{c}, p_{F}$ and $G_{c} / G_{F}$ can be expressed in terms of these parameters $\beta, \gamma, \rho_{F}, A R^{*}$ as

$$
\begin{gathered}
p_{c}=P_{c} / G_{c}=\gamma ; p_{F}=P_{F} / G_{F}=\beta \\
G_{c} / G_{F}=\frac{\rho_{F}}{A R^{*}} \frac{t_{[F]}}{1-\gamma^{2}}
\end{gathered}
$$

such that

$$
\chi=\operatorname{Re} \eta\left[\frac{1}{1-\gamma^{2}}+\frac{\rho_{F}}{A R^{*}} \frac{t_{[F]}}{\left(1-\beta^{2}\right)\left(1-\gamma^{2}\right)}\right]-1,
$$

which shows the explicit dependence of the NAMR on the thickness of the F-layer $t[F]$. From Eq. (3d) we obtain the following expression for $\operatorname{Re} \eta$

$$
\operatorname{Re} \eta=(1+\chi)\left(1-\gamma^{2}\right)\left[1+\frac{\rho_{F}}{A R^{*}} \frac{t_{[F]}}{1-\beta^{2}}\right]^{-1}
$$

So fitting NAMR data using Eq. 2a and Eq. (3c), would provide a way to obtain experimentally the mixing conductance $G^{\uparrow \downarrow}$. The dependence on $t_{[F]}$ reflects the spin-accumulation in the bulk ferromagnet. In the presence of spin-flip scattering, $t_{[F]}$ should be taken as the minimum of the geometrical layer thickness and the spin-flip diffusion length. This extra parameter can be eliminated in favor of measurable quantities by $\operatorname{expressing} A \Delta R^{T}=R^{T}(\theta=$ $\pi)-R^{T}(\theta=0)$ in terms of $\beta, \gamma, \rho_{F}, A R^{*}$ :

$$
A \Delta R^{T}=\frac{1}{G^{T}(\theta=\pi)}-\frac{1}{G^{T}(\theta=0)}=2 A R^{*} \gamma^{2} \frac{\left[1+\frac{\rho_{F}}{A R^{*}} \frac{t_{[F]}}{1-\beta^{2}}\left(\frac{\beta}{\gamma}\right)\right]^{2}}{\left[1+\frac{\rho_{F}}{A R^{*}} \frac{t_{[F]}}{1-\beta^{2}}\right]}
$$

If we assume that $(\beta / \gamma)=1\left(\right.$ For Py $/ \mathrm{Cu} / \mathrm{Py}: \beta_{\mathrm{Py}}=0.76, \gamma_{\mathrm{Py} / \mathrm{Cu}}=0.7,(\beta / \gamma)=1.08$ and for $\mathrm{CoFe} / \mathrm{Cu} / \mathrm{CoFe}$ $\left.\beta_{\mathrm{CoFe}}=0.65, \gamma_{\mathrm{CoFe} / \mathrm{Cu}}=0.75,(\beta / \gamma)=0.86\right)$ we can write

$$
\left[1+\frac{\rho_{F}}{A R^{*}} \frac{t_{[F]}}{1-\beta^{2}}\right]^{-1}=\frac{2 A R^{*} \gamma^{2}}{A \Delta R^{T}}
$$

and

$$
\operatorname{Re} \eta=(1+\chi)\left(1-\gamma^{2}\right) \frac{2 A R^{*} \gamma^{2}}{\Delta R^{T}}
$$

which gives an expression to determine $\operatorname{Re} G^{\uparrow \downarrow}$ without reference to the thickness parameter $t_{[F]}$.

In conclusion, the normalized angular magnetoresistance (NAMR) for CPP spin valves depends on the spin accumulation in the F-layers. This effect should be included in order to obtain reliable values of the mixing conductance by fitting experimental NAMR data 11 .

We thank W. P. Pratt for useful discussions. This work is part of the research program for the "Stichting voor Fundamenteel Onderzoek der Materie" (FOM).

[1] W.P. Pratt, Jr., S.-F. Lee, J.M. Slaughter, R. Loloee, P.A. Schroeder, and J. Bass, Phys. Rev. Lett. 66, 3060 (1991); M.A.M. Gijs, S. K. J. Lenczowski, J. B. Giesbers, Phys. Rev. Lett. 70 (1993) 3343; M.A.M. Gijs, G.E.W. Bauer, Adv. Phys. 46 (1997) 285; J. Bass, W. P. Pratt, J. Magn. Magn. Mater. 200 (1999) 274.

[2] J. C. Slonczewski, Phys. Rev. B 39, 6995 (1989); V. V. Ustinov and E. A. Kravtsov, J. Phys. : Cond. Matter 7, 3471 (1995); P. Dauguet, P. Gandit, J. Chaussy, S.F. Lee, A. Fert and P. Holody, Phys. Rev. B 54, 1083 (1996); H. E. Camblong, P.M. Levy and S. Zang, Phys. Rev. B 51, 16052 (1995); 
[3] A. Vedyayev, N. Ryzhanova, B. Dieny, P. Dauguet, P. Gandit and J. Chaussy, Phys. Rev. B 55, 3728 (1997).

[4] Yu.V. Nazarov, Phys. Rev. Lett. 73, 1420 (1994); Yu.V. Nazarov, Superlatt. and Microstruc. 25, 1221 (1999).

[5] A. Brataas, Yu. V. Nazarov, and G.E.W. Bauer, Phys. Rev. Lett. 84, 2481 (2000); A. Brataas, Yu. V. Nazarov, and G.E.W. Bauer, Eur. Phys. J. B 0: (0) 000-000 J 000 .

[6] D. Huertas-Hernando, Yu. V. Nazarov, A. Brataas and G.E.W. Bauer, Phys. Rev. B. 62, 5700 (2000).

[7] J. A. Katine, F. J. Albert, R. A. Buhrman, E. B. Myers and D. C. Ralph, Phys. Rev. Lett. 84, 3149 (2000).

[8] A. Fert and S.-F Lee, Phys. Rev. B 53, 6554 (1996).

[9] K.M. Schep, J.B.A.N. van Hoof, P.J. Kelly, G.E.W. Bauer, and J.E. Inglesfield, Phys. Rev. B56, 10805(1997).

[10] K. Kia, P. J. Kelly, G.E.W. Bauer and A. Brataas, unpublished.

[11] W. P. Pratt, private comunication.

\section{Figure Caption}

Fig. 1: Circuit model of a CPP spin valve, in term of reservoirs, resistors and nodes. Ferromagnetic layers (F) and metallic contact regions (C) are represented by resistors, characterized by spin conductance parameters. Two nodes connect each F-layer with a contact region $\mathrm{C}$. The third node represents the normal layer. Associated with each node is a $2 \times 2$ distribution matrix in spin space. The direction of the spin accumulation in each magnetic node (F-C node) is denoted by a unit vector $\overrightarrow{\mathbf{m}}$. The external reservoirs $(\mathrm{R})$ are supposed to be in local equilibrium. 


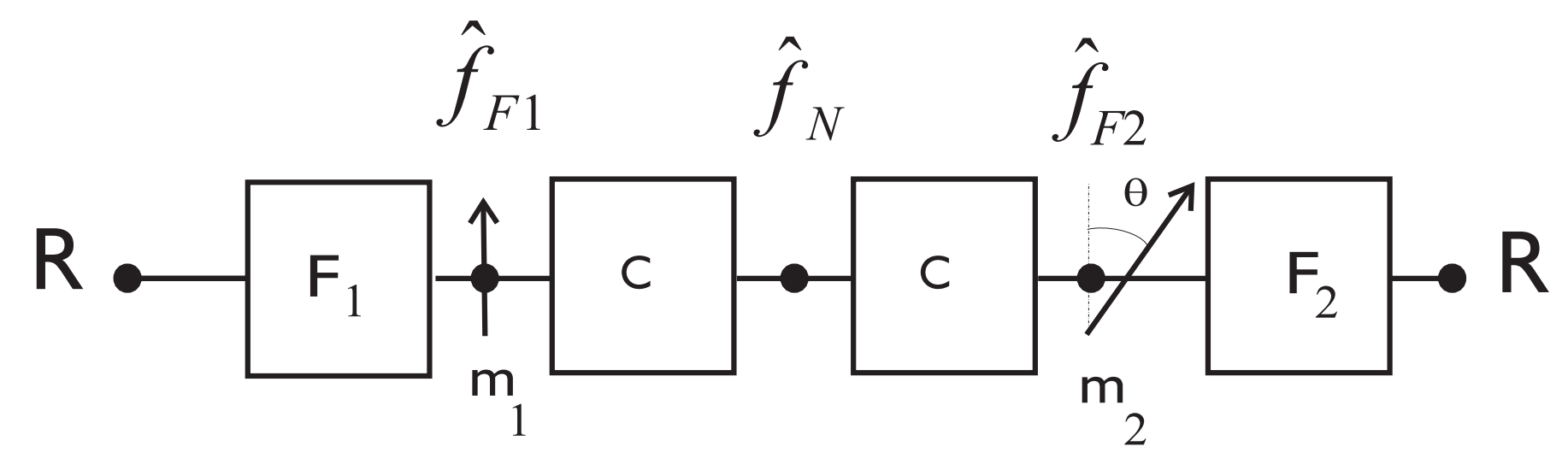

